\title{
Constraining the Structure of Sagittarius A*'s Accretion Flow with Millimeter Very Long Baseline Interferometry Closure Phases
}

\section{Citation}

Broderick, Avery E., Vincent L. Fish, Sheperd S. Doeleman, and Abraham Loeb. 2011. "CONSTRAINING THE STRUCTURE OF SAGITTARIUS A*'S ACCRETION FLOW WITH MILLIMETER VERY LONG BASELINE INTERFEROMETRY CLOSURE PHASES." The Astrophysical Journal 738 (1): 38. https://doi.org/10.1088/0004-637x/738/1/38.

\section{Permanent link}

http://nrs.harvard.edu/urn-3:HUL.InstRepos:41393157

\section{Terms of Use}

This article was downloaded from Harvard University's DASH repository, and is made available under the terms and conditions applicable to Other Posted Material, as set forth at http:// nrs.harvard.edu/urn-3:HUL.InstRepos:dash.current.terms-of-use\#LAA

\section{Share Your Story}

The Harvard community has made this article openly available.

Please share how this access benefits you. Submit a story.

\section{Accessibility}




\title{
CONSTRAINING THE STRUCTURE OF SAGITTARIUS A*'s ACCRETION FLOW WITH MILLIMETER VERY LONG BASELINE INTERFEROMETRY CLOSURE PHASES
}

\author{
Avery E. Broderick ${ }^{1}$, Vincent L. Fish ${ }^{2}$, Sheperd S. Doeleman ${ }^{2}$, and Abraham Loeb ${ }^{3}$ \\ ${ }^{1}$ Canadian Institute for Theoretical Astrophysics, 60 St. George Street, Toronto, ON M5S 3H8, Canada; aeb@ @ita.utoronto.ca \\ ${ }^{2}$ Massachusetts Institute of Technology, Haystack Observatory, Route 40, Westford, MA 01886, USA \\ ${ }^{3}$ Institute for Theory and Computation, Harvard University, Center for Astrophysics, 60 Garden Street, Cambridge, MA 02138, USA \\ Received 2011 June 10; accepted 2011 June 10; published 2011 August 10
}

\begin{abstract}
Millimeter wave very long baseline interferometry (mm-VLBI) provides access to the emission region surrounding Sagittarius A* (Sgr A*), the supermassive black hole at the center of the Milky Way, on sub-horizon scales. Recently, a closure phase of $0^{\circ} \pm 40^{\circ}$ was reported on a triangle of Earth-sized baselines (SMT-CARMA-JCMT) representing a new constraint upon the structure and orientation of the emission region, independent from those provided by the previously measured $1.3 \mathrm{~mm}$-VLBI visibility amplitudes alone. Here, we compare this to the closure phases associated with a class of physically motivated, radiatively inefficient accretion flow models and present predictions for future mm-VLBI experiments with the developing Event Horizon Telescope (EHT). We find that the accretion flow models are capable of producing a wide variety of closure phases on the SMT-CARMA-JCMT triangle and thus not all models are consistent with the recent observations. However, those models that reproduce the 1.3 mm-VLBI visibility amplitudes overwhelmingly have SMT-CARMA-JCMT closure phases between $\pm 30^{\circ}$, and are therefore broadly consistent with all current $\mathrm{mm}$-VLBI observations. Improving station sensitivity by factors of a few, achievable by increases in bandwidth and phasing together multiple antennas at individual sites, should result in physically relevant additional constraints upon the model parameters and eliminate the current $180^{\circ}$ ambiguity on the source orientation. When additional stations are included, closure phases of order $45^{\circ}-90^{\circ}$ are typical. In all cases, the EHT will be able to measure these with sufficient precision to produce dramatic improvements in the constraints upon the spin of Sgr A*.
\end{abstract}

Key words: accretion, accretion disks - black hole physics - Galaxy: center - submillimeter: general - techniques: interferometric

Online-only material: color figures

\section{INTRODUCTION}

It has recently become possible to study the emission regions of a handful of black holes on sub-horizon scales with millimeter wave very long baseline interferometry (mm-VLBI). Already, this technique has produced horizon-scale information on Sagittarius A* (Sgr A*), the $4.3 \times 10^{6} M_{\odot}$ black hole located at the center of the Milky Way (Ghez et al. 2008; Gillessen et al. 2009a, 2009b), using the Arizona Radio Observatory SubMillimeter Telescope (SMT) on Mount Graham, Arizona, James Clerk Maxwell Telescope (JCMT), and Sub-Millimeter Array (SMA) atop Mauna Kea in Hawaii, and the Combined Array for Research in Millimeter-wave Astronomy (CARMA) in Cedar Flat, California (Doeleman et al. 2008; Fish et al. 2011). Due to the limited signal to noise of these early experiments, they have produced primarily visibility amplitudes, related to the modulus of the Fourier transform of the intensity distribution of the source. Nevertheless, when analyzed in the context of physically motivated accretion flow models, they have resulted in dramatic constraints upon the spin orientation and magnitude (Broderick et al. 2009; Huang et al. 2009; Dexter et al. 2010; Broderick et al. 2011). However, the absence of phase information introduces fundamental degeneracies in the orientation of the modeled image and systematic uncertainties in the image structure generally.

The importance of visibility phase information has been appreciated since the beginning of radio interferometry. In the context of VLBI, it has only been widely possible since the introduction of "closure phases" and the associated development of self-calibration techniques (e.g., Jennison 1958; Jennison \& Latham 1959; Rogers et al. 1974; Pearson \& Readhead 1984; Thompson et al. 2001). The closure phases, which are equivalent to the argument of the bispectrum, are combinations of the visibility phases on baseline triangles (and thus VLBI station triples) that are insensitive to individual station-based phase errors, which otherwise typically dominate the phase uncertainties, e.g., due to pathlength variations from atmospheric turbulence. ${ }^{4}$ Specifically, if $\phi_{i j}$ is the visibility phase on the baseline between stations $i$ and $j$, the closure phase associated with three stations is

$$
\Phi_{i j k} \equiv \phi_{i j}+\phi_{j k}+\phi_{k i} .
$$

Even a handful of closure phases are diagnostic of the underlying image structure, e.g., the closure phases of a point source or Gaussian flux distribution (including asymmetric cases) are identically $0^{\circ}$, while that of an annulus may be either $0^{\circ}$ or $180^{\circ}$, depending upon the particular baselines considered. More general flux distributions produce non-vanishing closure phases, indicative of the symmetry of the image. Recently, Fish et al. (2011) reported the measurement of a closure phase at $1.3 \mathrm{~mm}$ for Sgr $\mathrm{A}^{*}$ of $0^{\circ} \pm 40^{\circ}$ on the SMT-JCMT-CARMA

\footnotetext{
4 Due to the degeneracy amongst possible triangles, given $N$ antennas there are only $(N-1)(N-2) / 2$ independent closure phases, and thus $N-1$ additional phases must be supplied to produce the full compliment of phase information. Frequently, these are obtained via self-calibration techniques, a combination of nonlinear algorithms in which the unknown phases are chosen such that the resulting image satisfies various physical constraints (see, e.g., Pearson \& Readhead 1984, for a detailed summary of self-calibration techniques).
} 
triangle. Note that this represents a new constraint upon models for the structure of the emitting region surrounding $\operatorname{Sgr} \mathrm{A}^{*}$, independent of those associated with the visibility amplitudes alone.

The Event Horizon Telescope (EHT) is a project underway that will extend current mm-VLBI arrays to shorter wavelengths $(0.8 \mathrm{~mm})$, increased sensitivity, and greater baseline coverage, substantially improving the ability of mm-VLBI to study black holes on Schwarzschild radius scales. (Doeleman et al. 2009a). Anticipated and potential future stations sites include Chile (Atacama Pathfinder Experiment, Atacama Submillimeter Telescope, and Atacama Large Millimeter Array; APEX, ASTE, and ALMA, respectively), Mexico (Large Millimeter Telescope; LMT), the South Pole (South Pole Telescope; SPT), and the IRAM telescopes in Spain (Pico Veleta; PV) and France (Plateau de Bure; PdB). Among these the longest baselines are $u \simeq 1.2 \times 10^{4} \mathrm{~km}$, corresponding to a maximum angular resolution of $\lambda / 2 u \simeq 11 \mu$ as at $230 \mathrm{GHz}(1.3 \mathrm{~mm})$ and $7.5 \mu$ as at $345 \mathrm{GHz}(0.87 \mathrm{~mm})$. Expected increases in bandwidth and the phasing together of elements within Hawaii, Chile, and CARMA (e.g., Weintroub 2008) will lead to substantial improvements in sensitivity. As a consequence, it will become possible in the near future to measure mm-VLBI closure phases on a variety of additional triangles with uncertainties considerably smaller than that of the Fish et al. (2011) result.

Here, motivated by previous efforts to model the $1.3 \mathrm{~mm}$ VLBI visibilities using physically motivated accretion models for $\mathrm{Sgr} \mathrm{A}^{*}$, we compute the closure phases implied by the radiatively inefficient accretion flow models and $1.3 \mathrm{~mm}-\mathrm{VLBI}$ visibility amplitude fits presented in Broderick et al. (2011). By doing so we address three immediate questions.

1. Is the new closure phase estimate consistent with the accretion flow models we have considered in particular and radiatively inefficient accretion flow models generally?

2. What is the strength of the constraint placed upon physically motivated accretion flow models and the estimates of black hole spin by the measured closure phase?

3. What are the strength of the constraints that will be possible in the near future as the EHT develops?

In Section 2, we briefly describe the accretion models and how we compute the closure phases. In Section 3, we compare the predicted closure phases with the measured values. In Section 4, we predict the closure phases for the EHT and compare these with the estimated uncertainties of the EHT. Finally, we summarize our conclusions in Section 6.

\section{COMPUTING ACCRETION FLOW CLOSURE PHASES}

\subsection{Accretion Modeling}

We model Sgr A*'s accretion flow as a radiatively inefficient accretion flow, the details of which may be found in Broderick et al. (2011), and references therein, and thus we only summarize the model here.

Sgr A* transitions from an inverted, presumably optically thick spectrum to an optically thin spectrum near millimeter wavelengths. This implies that near $1.3 \mathrm{~mm} \mathrm{Sgr} \mathrm{A*} \mathrm{is} \mathrm{only}$ becoming optically thin, and thus absorption in the surrounding medium is likely to be important. This transition does not occur isotropically, taking place at longer wavelengths for gas that is receding and at shorter wavelengths for gas that is approaching. Therefore, properly modeling the structure and relativistic radiative transfer is crucial to producing high fidelity images.
For concreteness, as in Broderick et al. (2011), we follow Yuan et al. (2003) and employ a model in which the accretion flow has a Keplerian velocity distribution, a population of thermal electrons with density and temperature

$$
n_{e, \text { th }}=n_{e, \text { th }}^{0}\left(\frac{r}{r_{\mathrm{S}}}\right)^{-1.1} e^{-z^{2} / 2 \rho^{2}}
$$

and

$$
T_{e}=n_{e}^{0}\left(\frac{r}{r_{\mathrm{S}}}\right)^{-0.84},
$$

respectively, and a toroidal magnetic field in approximate ( $\beta=10$ ) equipartition with the ions (which are responsible for the majority of the pressure), i.e.,

$$
\frac{B^{2}}{8 \pi}=\beta^{-1} n_{e, \text { th }} \frac{m_{p} c^{2} r_{\mathrm{S}}}{12 r} .
$$

In all of these, $r_{\mathrm{S}}=2 G M / c^{2}$ is the Schwarzschild radius, $\rho$ is the cylindrical radius, and $z$ is the vertical coordinate. Inside of the innermost-stable circular orbit we assume the gas is plunging upon ballistic trajectories. In principle the plunging gas can still radiate, though in practice it contributes little to the overall emission due to the large radial velocities it develops. In the case of the thermal quantities the radial structure was taken from Yuan et al. (2003), and the vertical structure was determined by assuming that the disk height is comparable to $\rho$. Note that all of the models we employ necessarily have the spin aligned with the orbital angular momentum of the accretion flow. For the regions that dominate the millimeter emission, this assumption is well justified due to disk precession and viscous torques, though it may be violated at large distances.

Thermal electrons alone are incapable of reproducing the nearly flat spectrum of $\operatorname{Sgr} A^{*}$ below $43 \mathrm{GHz}$. Thus, it is necessary to also include a nonthermal component. As with the thermal components, we adopt a self-similar model for a population of nonthermal electrons,

$$
n_{e, \text { nth }}=n_{e, \text { nth }}^{0}\left(\frac{r}{r_{\mathrm{S}}}\right)^{-2.02} e^{-z^{2} / 2 \rho^{2}},
$$

with a power-law distribution corresponding to a spectral index of 1.25 and cutoff below Lorentz factors of $10^{2}$ (consistent with Yuan et al. 2003). The radial power-law index was chosen to reproduce the low-frequency spectrum of $\mathrm{Sgr} \mathrm{A}^{*}$ and is insensitive to the black hole properties due to the distant location of the long-wavelength emission.

The primary emission mechanism at the wavelengths of interest is synchrotron radiation, arising from both the thermal and nonthermal electrons. We model the emission from the thermal electrons using the emissivity described in Yuan et al. (2003), appropriately altered to account for relativistic effects Broderick \& Blandford (see, e.g., 2004). Since we perform polarized radiative transfer via the entire complement of Stokes parameters, we employ the polarization fraction for thermal synchrotron as derived in Petrosian \& McTiernan (1983). In doing so, we have implicitly assumed that the emission due to thermal electrons is isotropic, which while generally not the case is unlikely to change our results significantly. For the nonthermal electrons, we follow Jones \& O'Dell (1977) for a powerlaw electron distribution, with an additional spectral break associated with the minimum electron Lorentz factor. For both emission components the absorption coefficients are determined 
directly via Kirchhoff's law. Images are then produced using the fully relativistic ray-tracing and radiative transfer schemes described in Broderick \& Loeb (2006a; 2006b) and Broderick (2006).

Because Yuan et al. (2003) neglected relativistic effects and assumed spherical symmetry, it is not directly applicable here. For these reasons, as in Broderick et al. (2011), the coefficients $\left(n_{e, \text { th }}^{0}, T_{e}^{0}, n_{e, \text { nth }}^{0}\right)$ were adjusted to fit the radio spectral energy distribution (SED) of Sgr A* (see Broderick et al. 2011, for details on the fitting procedure). We repeated this procedure for a large number of positions in the dimensionless spin-inclination $(a-\theta)$ parameter space, ${ }^{5}$ producing a tabulated set of the coefficients $\left(n_{e, \text { th }}^{0}, T_{e}^{0}, n_{e, \text { nth }}^{0}\right)$ at a large number of points throughout the $a-\theta$ parameter space. In all cases it was possible to fit the SED with extraordinary precision. From the tabulated values, the coefficients are then obtained at arbitrary $a$ and $\theta$ using high-order polynomial interpolation.

During the $1.3 \mathrm{~mm}-\mathrm{VLBI}$ observations Sgr A*'s flux varied by roughly $30 \%$. We model this as a variable accretion rate, moving the electron density normalization up and down. In practice, we reduced the electron density normalization by an amount sufficient to produce a total flux of $2.5 \mathrm{Jy}$, and then multiplied the resulting images by a correction factor during the mm-VLBI data analysis. Because the source is not uniformly optically thin, this is not strictly correct, though this makes a small change to the images themselves. We produced 9090 images, with flux normalized as described above, at $a \in\{0,0.01,0.02, \ldots, 0.98,0.99,0.998\}$ for each $\theta \in\left\{1^{\circ}, 2^{\circ}, \ldots, 89^{\circ}, 90^{\circ}\right\}$. We then produce models with arbitrary position angles, $\xi$, by rotating the images on the sky. For this purpose, we define $\xi$ as the position angle (east of north) of the projected spin vector.

\subsection{Computing the Closure Phases}

The intrinsic complex visibilities are obtained in the standard fashion:

$$
V(u, v)=\iint d \alpha d \beta e^{-2 \pi i(\alpha u+\beta v) / \lambda} I(\alpha, \beta),
$$

where $I(\alpha, \beta)$ is the intensity at a given set of angular coordinates. These are subsequently modified to account for the observed interstellar electron scattering (see, e.g., Bower et al. 2006; Broderick et al. 2011). ${ }^{6}$ The closure phase for a given triplet of observatories is then given by

$$
\Phi_{i j k}=\arg \left[V\left(u_{i j}, v_{i j}\right)\right]+\arg \left[V\left(u_{j k}, v_{j k}\right)\right]+\arg \left[V\left(u_{k i}, v_{k i}\right)\right] .
$$

We compute the $\Phi_{i j k}$ for all spin magnitude, inclination, and position angles considered in Broderick et al. (2011). From these we determine the probability density of a given $\Phi_{i j k}$ :

$$
p(\Phi)=\int d^{3} a p(\mathbf{a}) \delta\left[\Phi_{i j k}(\mathbf{a})-\Phi\right]
$$

\footnotetext{
5 The black hole angular momentum is given by $a G M^{2} / c$, with $a=1$ corresponding to a maximally rotating Kerr spacetime. We define $\theta$ such that models with $\theta=0^{\circ}$ are viewed along the spin axis, and thus the thick accretion disk viewed face-on. Conversely, models with $\theta=90^{\circ}$ are viewed perpendicular to the spin axis, and thus the thick accretion disk is viewed edge-on.

6 Note that the empirically measured Gaussian scattering kernel does not produce phase shifts in the visibilities, and thus we may neglect the scattering in computing the closure phases. Nevertheless, we must keep this effect for the computation of the expected closure phase uncertainties.
}

in which $p(\mathbf{a})$ is the probability of a given vector spin. For our purposes here we consider two forms of $p(\mathbf{a})$ : an isotropically distributed spin direction with a flat prior upon the spin magnitude, the same prior as that adopted in Broderick et al. (2011), and the posterior probability of a given spin, defined in Broderick et al. (2011), after fitting the $1.3 \mathrm{~mm}$-VLBI amplitudes. We will refer to these as the "isotropic" and "amplitude-fitted" priors, respectively. Interpolation errors associated with the finite density of points in the spin parameter space and the finite size of the imaged region, resulted in some small-amplitude saw-tooth features in the $p(\Phi)$. As a consequence, we smoothed the $p(\Phi)$ shown on scales of $1^{\circ}-9^{\circ}$, depending upon the particular case, with $5^{\circ}$ being typical. Where large spike features appeared we did no smoothing, and in all cases the wings of the probability distribution remained unchanged.

Note that fitting the visibility amplitudes necessarily imparts a $180^{\circ}$ degeneracy in the position angle, corresponding to a sign change in the resulting closure phase. As a result, in principle, all closure phase estimates we present are unique only up to a sign. Nevertheless, we show the closure phases associated with one particular choice for the position angle, specifically that for which the spin of the most likely configuration is oriented $-52^{\circ}$ east of north.

\subsection{Closure Phase Uncertainties}

The precision with which a given closure phase may be measured depends upon the signal-to-noise ratio $(\mathrm{S} / \mathrm{N})$ of the visibilities on the individual baselines, $s_{i j} \equiv V_{i j} \sqrt{2 B \tau / \mathrm{SEFD}_{i} \mathrm{SEFD}_{j}}$, where $V_{i j}$ is the visibility amplitude, $B$ is the bandwidth, $\tau$ is the atmospheric coherence time, and $\mathrm{SEFD}_{i}$ is the system equivalent flux density for a given station. Here we take $\tau=10 \mathrm{~s}$, typical of the atmospheric coherence times at the wavelengths and sites of interest. However, because closure phases are insensitive to atmospheric phase fluctuations, they may be coherently averaged over timescales much larger than $\tau$, with the measurement uncertainties decreasing as $\sqrt{\tau / T}$. This is limited by the timescales on which the baseline orientations and the intrinsic structure of Sgr A* change significantly. In quiescence, the former is the limiting factor, and thus we choose $T=10$ minutes. However, during periods of flaring activity, or if Sgr A* exhibits some dynamical structure, this could be considerably shorter. Finally, unless otherwise stated, we assume $B=4 \mathrm{GHz}$, the anticipated near-term bandwidth target of the EHT, and adopt the $\mathrm{SEFD}_{i}$ reported in Table 1 of Doeleman et al. (2009b), partially reproduced in Table 1 here. $^{7}$ Specifically we assume that the SMA, CSO, and JCMT are phased together in Hawaii, six of the $10.4 \mathrm{~m}$ and two of the $6 \mathrm{~m}$ CARMA antennas are phased together, and ten of the $12 \mathrm{~m}$ ALMA antennas are phased together in Chile. In terms of the $s_{i j}$, the closure phase uncertainty is given by

$$
\sigma_{\Phi_{i j k}}=\sqrt{\frac{\tau}{T}} L^{-1}\left[L\left(s_{i j}\right) L\left(s_{j k}\right) L\left(s_{k i}\right)\right],
$$

where

$$
L(s) \equiv \sqrt{\frac{\pi}{8}} s e^{-s^{2} / 4}\left[I_{0}\left(\frac{s^{2}}{4}\right)+I_{1}\left(\frac{s^{2}}{4}\right)\right]
$$

\footnotetext{
7 This choice of bandwidth and set of $\mathrm{SEFD}_{i}$ results in baseline $\mathrm{S} / \mathrm{Ns}$ approximately an order of magnitude larger than those obtained during the 1.3 mm-VLBI observations reported in Fish et al. (2011).
} 


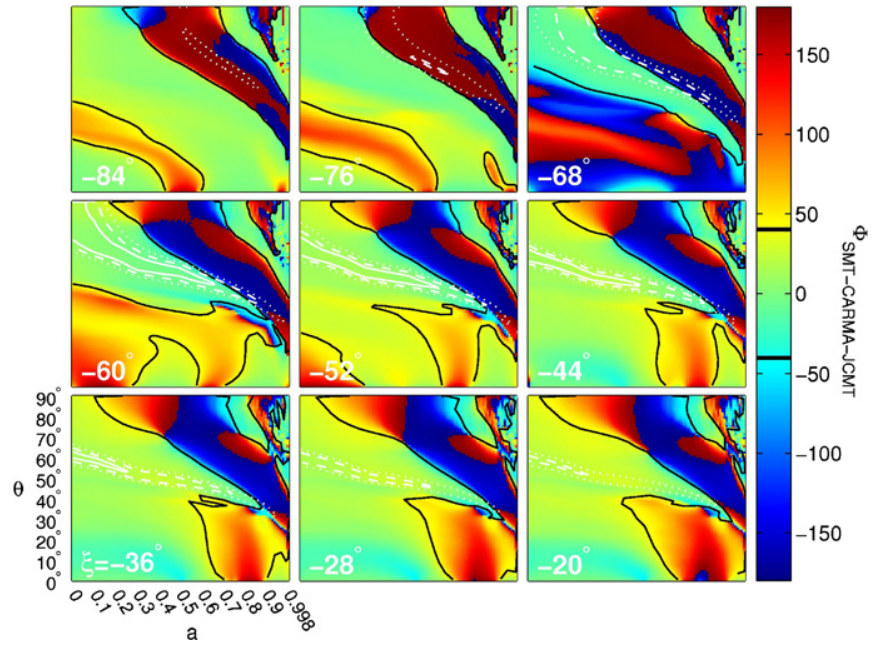

Figure 1. SMT-CARMA-JCMT closure phases, determined at 12:20 UTC on Day 96 of 2009, for the accretion models considered by Broderick et al. (2011) as a function of dimensionless spin magnitude $(a)$ and inclination $(\theta)$ for a number of position angles $(\xi)$. Black contour lines show the closure phase limit of $0^{\circ} \pm 40^{\circ}$ reported by Fish et al. (2011). For reference, the posterior probability contours (white) associated with the $1 \sigma$ (solid), $2 \sigma$ (dashed), and $3 \sigma$ (dotted) cumulative probabilities as determined in Broderick et al. (2011) are overlaid. Note that these are degenerate to $\Phi \rightarrow-\Phi$.

(A color version of this figure is available in the online journal.)

Table 1

Estimated System Equivalent Flux Densities at $230 \mathrm{GHz}$

\begin{tabular}{lccc}
\hline \hline Facility & $N^{\mathrm{a}}$ & $\begin{array}{c}\text { Diameter }^{\mathrm{a}} \\
(\mathrm{m})\end{array}$ & $\begin{array}{c}\text { SEFD }^{\mathrm{b}} \\
(\mathrm{Jy})\end{array}$ \\
\hline Hawaii & 8 & 23 & 4,900 \\
CARMA & 8 & 27 & $6,500^{\mathrm{c}}$ \\
SMT & 1 & 10 & 11,900 \\
LMT & 1 & 32 & $10,000^{\mathrm{d}}$ \\
APEX & 1 & 12 & 6,500 \\
ALMA & 10 & 38 & 500 \\
\hline
\end{tabular}

Notes.

${ }^{a}$ Effective aperture when number of antennas $(N)$ are phased together.

${ }^{\mathrm{b}}$ Expected system equivalent flux density values toward Sgr A* include typical weather conditions and opacities.

${ }^{c}$ Based upon recent observations.

${ }^{\mathrm{d}}$ Completion of the dish and upgrades to the surface accuracy and receiver will eventually lower the SEFD by more than a factor of 10 .

in which $I_{0}$ and $I_{1}$ are hyperbolic Bessel functions of the first kind (Rogers et al. 1984, 1995). In the high $\mathrm{S} / \mathrm{N}$ limit $\left(s_{i j}, s_{j k}, s_{k i} \gg 1\right)$ this reduces to

$$
\sigma_{\Phi_{i j k}} \simeq \sqrt{\frac{\tau}{T}}\left[s_{i j}^{-2}+s_{j k}^{-2}+s_{k i}^{-2}\right]^{-1 / 2},
$$

though we will make use of the exact expression. Note that since $\sigma_{\Phi}$ depends upon the the individual $\mathrm{S} / \mathrm{N}$ values, it is model dependent; the values we quote are associated with the most likely accretion model, as determined via the $1.3 \mathrm{~mm}$-VLBI visibility amplitudes alone.

\section{CLOSURE PHASE ON THE SMT-CARMA-JCMT TRIANGLE: COMPARISON WITH MEASURED VALUES}

There are two 15 minute periods over which Sgr A*'s closure phase was measured on the SMT-JCMT-CARMA triangle on the night of 2009 April 6 (corresponding to day 96), beginning on 12:20 UTC and 12:40 UTC (Fish et al. 2011). The distribution

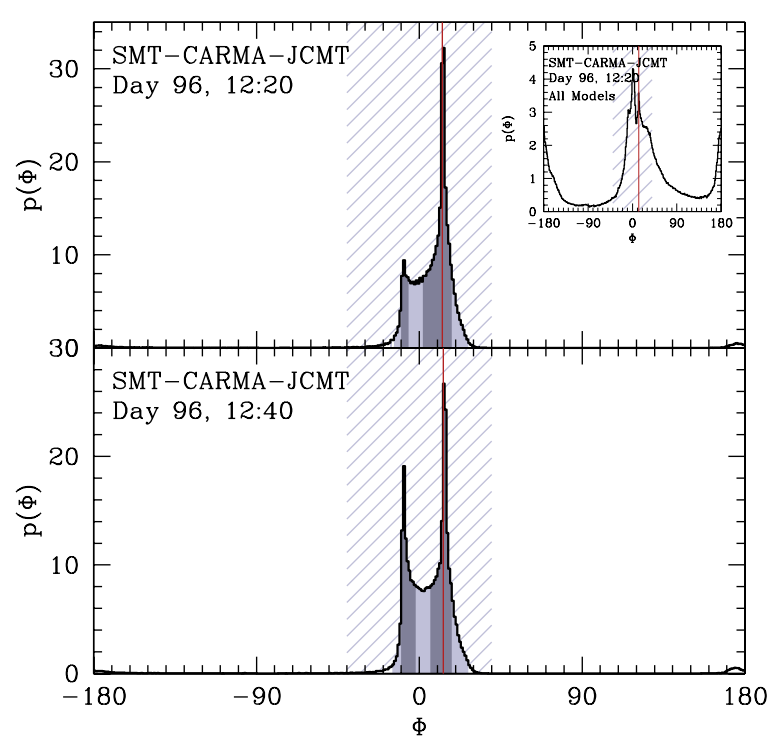

Figure 2. Probability distribution of the closure phases associated with the accretion flow models considered by Broderick et al. (2011) for the SMT-JCMT-CARMA triangle during the times at which Fish et al. (2011) report measurements of this quantity. The dark gray and light gray regions are the $1 \sigma$ and $2 \sigma$ regions, defined by cumulative probability, and the light gray hatched region shows the measured value $\left(0^{\circ} \pm 40^{\circ}\right)$. The vertical red line indicates the closure phase associated with the most likely accretion flow model. For reference, the distribution of closure phases when the $1.3 \mathrm{~mm}-\mathrm{VLBI}$ prior is not applied is shown in the upper inset. Note that these are degenerate to $\Phi \rightarrow-\Phi$

(A color version of this figure is available in the online journal.)

of the closure phases at 12:20 UTC in spin magnitude $(a)$, spin inclination $(\theta)$, and spin position angle $(\xi)$ are shown in Figure 1, with the closure phases at 12:40 UTC differing by only a small amount. For reference the $1 \sigma, 2 \sigma$, and $3 \sigma$ posterior probability contours from the $1.3 \mathrm{~mm}$-VLBI visibility fits obtained in Broderick et al. (2011) are overlaid, shown by the solid, dashed, and dotted white contours, respectively.

It is immediately apparent that small closure phases are not generic. Assuming the isotropic prior, fewer than $49 \%$ of the accretion flow models are consistent with the closure phase measurement. The inset in Figure 2 bears this out; while small closure phases are marginally preferred, a large closure phase island exists with a substantial likelihood of any phase being measured. The excluded models are not confined to a particular region in the spin parameter space, extending over a wide range of spin magnitudes, inclinations, and position angles. Thus, despite the large uncertainties, the measured closure phase has considerable diagnostic ability.

However, this changes dramatically when we restrict our attention to those models which are consistent with the $1.3 \mathrm{~mm}$-VLBI amplitudes. The white contours in Figure 1 show the posterior probability of a given spin vector based upon the $1.3 \mathrm{~mm}$-VLBI visibility amplitudes alone, i.e., the amplitudefitted prior. The regions of high probability are confined to a narrow valley of low closure phases. Hence, while accretion models generally are not necessarily consistent with the observed closure phases, the vast majority of those that were found to reproduce the visibility amplitudes are broadly consistent with the recent measurement. Once the amplitude-fitted prior is adopted, the probability of finding a closure phase that is larger than $40^{\circ}$ falls to less than $3 \%$.

The amplitude-fitted models that violate the recent closure phase measurement are confined to the $2 \sigma$ significance regions, 

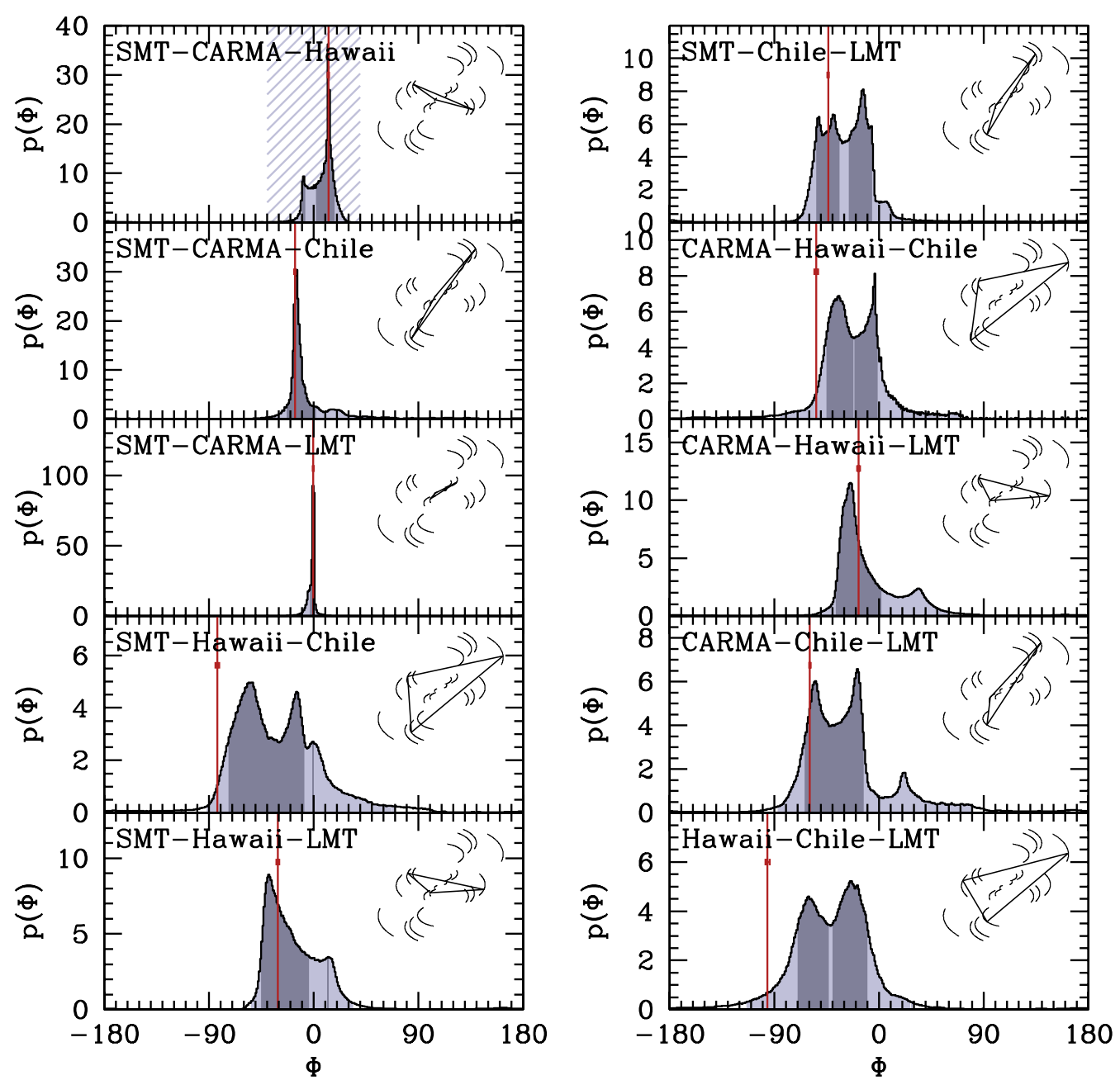

Figure 3. Probability distributions for the closure phases at 12:20 UTC on day 96 of 2009 associated with the accretion flow models considered by Broderick et al. (2011) for all triangles constructed from the SMT, Hawaii, CARMA, LMT, and Chile stations. The hatched region in the upper left indicates the closure phase measurement reported in Fish et al. (2011). In all plots the dark and light gray regions are the $1 \sigma$ and $2 \sigma$ regions, defined by cumulative probability, the vertical red lines show the closure phase of the most likely accretion flow model, and the red horizontal error bar indicates the accuracy with which the EHT should be able to measure the closure phases in the future on 10 minute timescales (using 10 ALMA antennas in Chile, employing the recently phased sites in Hawaii, and 8 CARMA antennas). For reference the $u-v$ tracks are shown in the upper right of each plot, with the triangle for the closure phase plotted indicated explicitly. Note that these are degenerate to $\Phi \rightarrow-\Phi$.

(A color version of this figure is available in the online journal.)

primarily at large spin magnitude and large negative position angles, where the closure phase is $\sim 180^{\circ}$. This behavior is similar to that exhibited by annular models, in which the closure phase varies between $0^{\circ}$ and $180^{\circ}$, depending upon the angular size of the annulus. As a result, improvements of factors of a few in sensitivity will allow closure phase measurements that can compete with the visibility amplitudes in their power to constrain the structure of Sgr A*.

These conclusions are borne out by the closure phase probability distributions shown in Figure 2, computed with the amplitude-fitted prior. For these we show both times, which exhibit slightly different distributions, though in both cases the closure phases are dominated by peaks near $-10^{\circ}$ and $10^{\circ}$. The probability of measuring a closure phase within the allowed range exceeds $97 \%$ at both times. The closure phase of the model with the highest amplitude-fitted prior probability (based upon the $1.3 \mathrm{~mm}$-VLBI visibility amplitude) is located at 12.6 . However, significant probabilities extend out to $\Phi \simeq \pm 30^{\circ}$, and thus factor of few improvements in sensitivity necessarily result in a significant likelihood of detecting non-trivial closure phases.

\section{CLOSURE PHASES ON FUTURE TRIANGLES}

The development of the EHT will be characterized by the inclusion of additional baselines and improvements in station sensitivity. Thus, we also compute the closure phases along triangles including intermediate and large north-south baselines. Figure 3 shows the probability distribution for closure phases on all triangles made with the SMT, CARMA, Hawaii, the LMT, and Chile, as seen at 12:20 UTC on day 96 of 2009. Closure phase distributions on other days and times are qualitatively similar. While the closure phase on the SMT-CARMA-Hawaii triangle is small, this is not generic. Closure phases with magnitudes of $\sim 45^{\circ}$ are likely on five of ten triangles, including the SMT-Hawaii-Chile and Hawaii-Chile-LMT triangles, for which the most probable model has closure phases comparable to $-90^{\circ}$ (shown by the red vertical lines in each panel). ${ }^{8}$

\footnotetext{
8 Note that in $30 \%$ of the cases shown the red line lies outside the $1 \sigma$ region. This is not unexpected given the definition of the $1 \sigma$ and $2 \sigma$ regions, and does not indicate that the model with the highest posterior probability is inherently unlikely. These deviations are generally largest for large open triangles, associated with the intrinsically larger value and distribution of closure phases in these cases.
} 

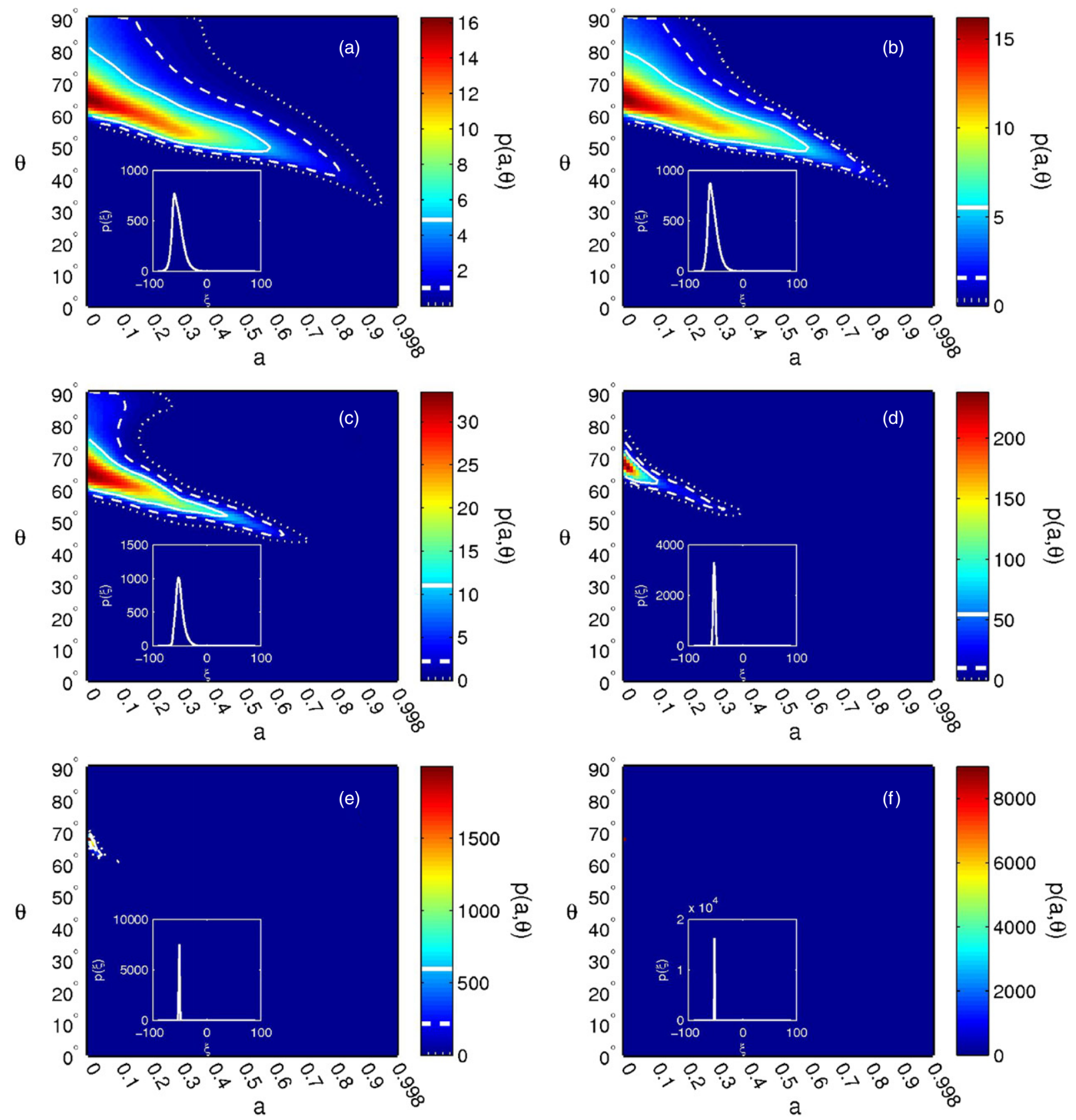

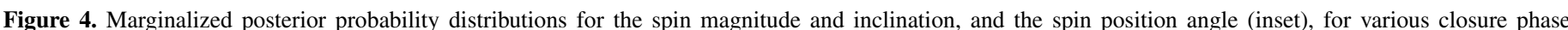

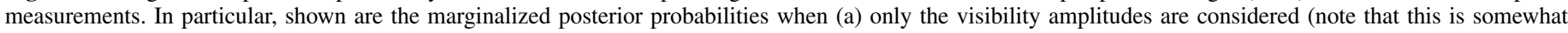

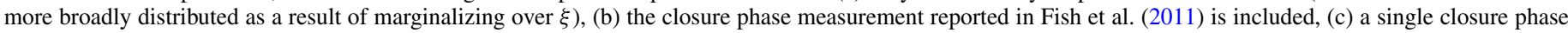

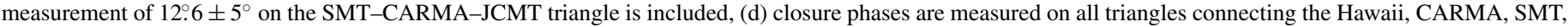

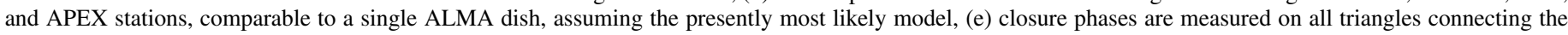

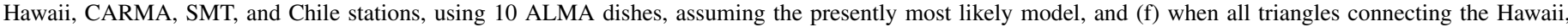

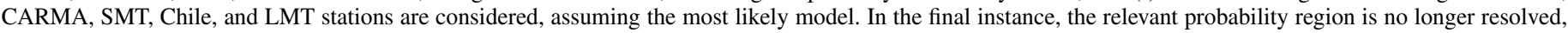
dominated overwhelmingly by a single pixel. See the text for more details.

(A color version of this figure is available in the online journal.)

More importantly, with improvements in the station sensitivity, arising from increases in bandwidth and the phasing together of multiple elements at individual stations, substantial improvements in the precision with which closure phases can be measured are possible. Despite the small visibilities associated with the most probable model (see Broderick et al. 2011), on all baselines constructed from the five stations we consider, Sgr A* is detectable on the typical atmospheric coherence time $(10 \mathrm{~s})$ with $\mathrm{S} / \mathrm{N}$ exceeding unity. Over the dynamical timescales of Sgr A*, roughly 10 minutes, the uncertainty in the closure phase is re- duced by an additional order of magnitude, resulting in the red, horizontal error bars in the individual panels of Figure 3, ranging from 0.6 to 1.8 . In all cases this is much smaller than the presently allowed range of values and provides sufficient precision to distinguish likely values from zero.

\section{IMPROVING THE CONSTRAINTS UPON Sgr A*'s SPIN}

The high precision with which closure phases may be measured in the near future, warrants a brief examination of their ability to help constrain the spin of Sgr A*. To estimate this we 
consider the effects of including a single closure phase measurement on each of the various station triangles discussed in Section 4 . In practice, where many closure phase measurements for each triangle are anticipated, these are expected to be even more constraining.

Figure 4(a) shows the posterior probability distribution obtained from analyzing the $1.3 \mathrm{~mm}$-VLBI amplitudes alone, as a function of spin magnitude $(a)$ and inclination $(\theta)$, after marginalized over the spin position angle $(\xi) .{ }^{9}$ Including the closure phase measurement reported in Fish et al. (2011) eliminates some of the high-spin, high-inclination models, producing the probability distribution seen in Figure 4(b). Nevertheless, the $1 \sigma$ and $2 \sigma$ contours are not significantly affected. This represents the present constraints upon the spin of Sgr A*.

To illustrate the improvement associated with higherprecision measurements and additional triangles, we will assume the most likely model, as defined by the $1.3 \mathrm{~mm}$-VLBI visibility amplitudes alone, is the "true" model for Sgr A*'s emission region. This allows us to compute the visibilities throughout the $u-v$ plane, obtain the $\mathrm{S} / \mathrm{N}$ at each point, and thus infer both the expected closure phase, $\Phi_{i j k, 0}$ and the precision with which it may be measured, $\sigma_{\Phi_{i j k, 0}}$. Based upon this, we can construct a likelihood associated with these observations,

$$
\mathcal{L}(\mathbf{a})=C \mathcal{L}_{|\mathrm{V}|}(\mathbf{a}) \exp \left[-\sum_{i j k} \frac{\left[\Phi_{i j k, 0}-\Phi_{i j k}(\mathbf{a})\right]^{2}}{2 \sigma_{\Phi_{i j k, 0}}^{2}}\right],
$$

where $C$ is some constant and $\mathcal{L}_{|\mathrm{V}|}$ is the likelihood of observing the measured visibility amplitudes alone. This may then be used to produce a posterior probability density, $p(\mathbf{a})$ ), as described in Broderick et al. (2011), the particulars of which depend upon which closure phase measurements we choose to include and their associated measurement uncertainties. In what follows we consider a handful of possible measurements that are relevant for upcoming observations.

Improving the precision of the closure phase measurement on the SMT-CARMA-JCMT triangle to $\pm 5^{\circ}$ substantially improves the spin estimation in two respects. First, it would conclusively eliminate the $180^{\circ}$ degeneracy in the position angle resulting from fitting visibility amplitudes alone. Second, as seen in Figure 4(c), it begins to significantly modify the $1 \sigma$ and $2 \sigma$ contours. Thus, even with modest precisions, closure phase measurements can substantial improve black hole spin estimation in Sgr A*.

For comparison with ongoing observations, we show in Figure 4(d) the constraints associated with closure phases measured with a phased Hawaii, phased CARMA, SMT, and APEX, all with a $1 \mathrm{GHz}$ bandwidth (in contrast to the $4 \mathrm{GHz}$ bandwidth assumed elsewhere). Even without the substantial sensitivity improvements associated with large bandwidths and phasing up multiple ALMA antennas, the resulting constraint is strengthened dramatically, in principle allowing a spin magnitude estimate with precision \pm 0.1 .

The spin constraints associated with observations with a phased ALMA are shown in Figure 4(e), at which point the allowed model parameter space becomes too small to resolve with the parameter sampling we employed. When the LMT is included (Figure 4(f)), the allowed region is reduced to a single grid point in our model space $(\Delta a=0.01, \Delta \theta=$ $\left.\Delta \xi=1^{\circ}\right)$. Whether such high-precision spin estimation is

\footnotetext{
9 Note that the allowed region is somewhat broader than shown in Figure 1 due to this marginalization.
}

possible in practice is almost certainly going to be limited by the systematic uncertainties in the modeling of the emission region. Nevertheless, the constraining power of even a handful of closure phase measurements is clear.

\section{CONCLUSIONS}

The measurement of mm-VLBI closure phases in Sgr A* provides an independent, significant constraint upon the structure of the emission region. Models based upon radiatively inefficient accretion flows do not generally have small closure phases and thus are not generally consistent with the recently measured value on the SMT-CARMA-JCMT triangle. As a consequence, even in the presence of large uncertainties, closure phases can place significant constraints upon the structure of Sgr A*.

However, when only accretion flow models that fit the $1.3 \mathrm{~mm}$-VLBI visibility amplitudes are considered, the probability of finding an excluded closure phase falls to less than $3 \%$. Hence, the vast majority of the acceptable models in Broderick et al. (2011) are broadly consistent with all current $1.3 \mathrm{~mm}-\mathrm{VLBI}$ constraints. This is surprising since the $1.3 \mathrm{~mm}$ VLBI amplitudes and closure phase measurements provide independent constraints, and thus having fit the accretion models to the former there is no a priori reason to expect that they would be consistent with the latter. Based upon the models that are consistent with the $1.3 \mathrm{~mm}$-VLBI visibility amplitudes, we predict that the closure phase on the SMT-CARMA-JCMT triangle during the time it was measured was between $\pm 30^{\circ}$, with $\pm 13^{\circ}$ most preferred. Improving the $\mathrm{S} / \mathrm{N}$ of $\mathrm{mm}-\mathrm{VLBI}$ observations by a factor of a few, achievable by phasing up multiple CARMA antennas and employing the recently phased sites in Hawaii, should allow detection of a non-zero closure phase on the SMT-CARMA-JCMT triangle. Note that this would eliminate the existing $180^{\circ}$ ambiguity in the orientation of Sgr A*.

If $\mathrm{Sgr} \mathrm{A}^{*}$ is well described by radiatively inefficient accretion flow models, currently acceptable closure phases on large triangles (e.g., SMT-Hawaii-Chile and Hawaii-Chile-LMT) can be as large as $90^{\circ}$, and typical values on triangles which include long baselines of $45^{\circ}$. Future improvements in sensitivity associated with the near-term development of the EHT will substantially increase the precision with which closure phases can be measured. On all triangles we considered (constructed from the SMT, CARMA, Hawaii, the LMT, and Chile) the resulting precision should be sufficient to measure the deviations from $0^{\circ}$.

Improved closure phase measurements and baseline coverage result in dramatically improved constraints upon the spin of Sgr A*. Including a single antenna in Chile (e.g., APEX or a single ALMA dish) produces spin estimates with precisions of roughly \pm 0.1 . With a partially phased ALMA station, this improves to \pm 0.03 , and with the LMT is better than \pm 0.01 , at which point we fail to resolve the likely region. In the latter cases, the spin estimation will almost certainly be limited by systematic uncertainties in the modeling of the emission region motivating the consideration of more sophisticated accretion and outflow models.

This work was supported in part by NSF grants AST0907890, AST-0807843, and AST-0905844, and NASA grants NNX08AL43G and NNA09DB30A. A.E.B. gratefully acknowledges the support of the Beatrice D. Tremaine Fellowship. 


\section{REFERENCES}

Bower, G. C., Goss, W. M., Falcke, H., Backer, D. C., \& Lithwick, Y. 2006, ApJ, 648, L127

Broderick, A. E. 2006, MNRAS, 366, L10

Broderick, A., \& Blandford, R. 2004, MNRAS, 349, 994

Broderick, A. E., Fish, V. L., Doeleman, S. S., \& Loeb, A. 2009, ApJ, 697, 45

Broderick, A. E., Fish, V. L., Doeleman, S. S., \& Loeb, A. 2011, ApJ, 735, 110

Broderick, A. E., \& Loeb, A. 2006a, ApJ, 636, L109

Broderick, A. E., \& Loeb, A. 2006b, MNRAS, 367, 905

Dexter, J., Agol, E., Fragile, P. C., \& McKinney, J. C. 2010, ApJ, 717, 1092

Doeleman, S. S., Fish, V. L., Broderick, A. E., Loeb, A., \& Rogers, A. E. E. 2009a, ApJ, 695, 59

Doeleman, S. S., et al. 2008, Nature, 455, 78

Doeleman, S. S., et al. 2009b, in Astronomy, Vol. 2010, Astro2010: The Astronomy and Astrophysics Decadal Survey, Science White Papers, 68

Fish, V. L., et al. 2011, ApJ, 727, L36

Ghez, A. M., et al. 2008, ApJ, 689, 1044
Gillessen, S., Eisenhauer, F., Fritz, T. K., Bartko, H., Dodds-Eden, K., Pfuhl, O., Ott, T., \& Genzel, R. 2009a, ApJ, 707, L114

Gillessen, S., Eisenhauer, F., Trippe, S., Alexander, T., Genzel, R., Martins, F., \& Ott, T. 2009b, ApJ, 692, 1075

Huang, L., Takahashi, R., \& Shen, Z. 2009, ApJ, 706, 960

Jennison, R. C. 1958, MNRAS, 118, 276

Jennison, R. C., \& Latham, V. 1959, MNRAS, 119, 174

Jones, T. W., \& O’Dell, S. L. 1977, ApJ, 214, 522

Pearson, T. J., \& Readhead, A. C. S. 1984, ARA\&A, 22, 97

Petrosian, V., \& McTiernan, J. M. 1983, Phys. Fluids, 26, 3023

Rogers, A. E. E., Doeleman, S. S., \& Moran, J. M. 1995, AJ, 109, 1391

Rogers, A. E. E., Moffet, A. T., Backer, D. C., \& Moran, J. M. 1984, Radio Sci., 19,1552

Rogers, A. E. E., et al. 1974, ApJ, 193, 293

Thompson, A. R., Moran, J. M., \& Swenson, G. W., Jr. 2001, Interferometry and Synthesis in Radio Astronomy, (2nd ed.; Weinheim: Wiley)

Weintroub, J. 2008, J. Phys. Conf. Ser., 131, 012047

Yuan, F., Quataert, E., \& Narayan, R. 2003, ApJ, 598, 301 